\title{
Generic theory of colloidal transport
}

\author{
Frank Jülicher ${ }^{1}$ and Jacques Prost ${ }^{2,3}$ \\ ${ }^{1}$ Max-Planck Institute for the Physics of Complex Systems, \\ Nöthnitzerstr. 38, 01187 Dresden, Germany \\ ${ }^{2}$ ESPCI, 10 rue Vauquelin, 75231 Paris Cedex 05, France \\ ${ }^{3}$ Physicochimie Curie, Institut Curie, \\ 26 rue d'Ulm, 75231 Paris Cedex 05, France
}

\begin{abstract}
We discuss the motion of colloidal particles relative to a two component fluid consisting of solvent and solute. Particle motion can result from (i) net body forces on the particle due to external fields such as gravity; (ii) slip velocities on the particle surface due to surface dissipative phenomena. The perturbations of the hydrodynamic flow field exhibits characteristic differences in cases (i) and (ii) which reflect different patterns of momentum flux corresponding to the existence of net forces, force dipoles or force quadrupoles. In the absence of external fields, gradients of concentration or pressure do not generate net forces on a colloidal particle. Such gradients can nevertheless induce relative motion between particle and fluid. We present a generic description of surface dissipative phenomena based on the linear response of surface fluxes driven by conjugate surface forces. In this framework we discuss different transport scenarios including self-propulsion via surface slip that is induced by active processes on the particle surface. We clarify the nature of force balances in such situations.
\end{abstract}




\section{INTRODUCTION}

Motion of colloidal particles immersed in a fluid can be driven by gradients of concentration or pressure even in the absence of net body forces on the particle. In such situations, particle motion results from relative motion between particle and fluid induced by surface slip [1]. As a result, the particle moves relative to the fluid without net force.

Physical mechanisms that underlie colloidal transport in a fluid can also be used to drive the swimming of self-propelling particles. Recently, several scenarios have been discussed by which colloidal objects can self-propel. In the case of self-electrophoresis, swimming in a fluid is driven by self-generated electric dipole fields, acting at the fluid particle interface [2, 3]. Phoretic swimmers are driven by a chemical reaction that is catalyzed in an asymmetric manner on the particle surface [4, 5, 6]. The surface reaction generates a concentration field near the particle which is asymmetric. The propulsion results from surface slip generated by a local concentration gradient at the particle surface. Active processes on a surface also propel many cells and microorganisms. An important example is motion driven by motile

cilia and other active cellular processes [7]. In a coarse-grained description, the beating motion of many cilia on a surface effectively generates surface slip velocities that drive propulsion [8, 9].

Here, we discuss a general theoretical framework to describe the motion of colloidal particles in a systematic and controlled way. Our hydrodynamic description is based on conservation laws and linear irreversible thermodynamics in a two-component fluid. Extension to multicomponent fluids is straightforward. The basic concepts discussed here are well established [10, 11, 12]. However, some confusion in the literature calls for clarification [13].

In section II, we review the general hydrodynamic equations of a two-component fluid. We express the conservation laws for mass, energy and momentum and identify the conjugate fluxes and forces. The hydrodynamic equations follow from a description of dissipative fluxes driven by conjugate thermodynamic forces. In section III, we discuss conditions for which concentration gradients and pressure gradients can be generated and the time scales during which they persist. A generic theory of dissipative processes associated with slip velocities on a solid surface that is in contact with a two-component fluid is discussed in section IV. Section $\mathrm{V}$ describes the motion of a colloidal particle in pressure gradients and concentration gradients. Self-propulsion of a colloidal object due to self-generated concentration gradients 
as well as due to active surface processes are discussed in section VI. We conclude our work with a discussion.

\section{HYDRODYNAMICS OF A TWO COMPONENT FLUID}

In order to define all basic concepts clearly, we first review the hydrodynamics of a twocomponent fluid characterized by a hydrodynamic flow field and diffusive fluxes. Using the systematic formulation of hydrodynamic equations based on irreversible thermodynamics [10], we follow the discussion presented in [14]. The fluid consists of solvent $a$ and solute $b$ with concentrations (numbers of molecules per unit volume) $n_{a}$ and $n_{b}$. The molecular masses of the two components are $m_{a}$ and $m_{b}$, respectively. We consider the incompressible case where the molecular volumes $v_{a}$ and $v_{b}$ are constant.

Conservation laws and entropy production. The molecular concentrations satisfy the conservation laws

$$
\frac{\partial n_{i}}{\partial t}+\nabla \cdot \mathbf{J}_{i}=0
$$

with $i=a, b$, where $\mathbf{J}_{i}$ denote particle currents. The mass density of the fluid is $\rho=$ $(1-\phi) m_{a} / v_{a}+\phi m_{b} / v_{b}$. Here $\phi=n_{b} v_{b}$ is the volume fraction of the solute. Incompressibility implies that $n_{a} v_{a}+n_{b} v_{b}=1$. Mass conservation can be expressed as

$$
\frac{\partial \rho}{\partial t}+\nabla \cdot(\rho \mathbf{v})=0
$$

where the hydrodynamic flow velocity $\mathbf{v}$ is the velocity of the center of mass of local volume elements. The particle currents can be decomposed in a center of mass flux and a relative flux $\mathbf{j}=\mathbf{j}_{\mathbf{b}}=-\mathbf{j}_{\mathbf{a}}$ :

$$
\mathbf{J}_{i}=n_{i} \mathbf{v}+\frac{\mathbf{j}_{i}}{m_{i}}
$$

Momentum conservation is described by the balance equation for the momentum density $\rho \mathbf{v}$

$$
\partial_{t}\left(\rho v_{\alpha}\right)-\partial_{\beta} \sigma_{\alpha \beta}=-\rho g \delta_{\alpha z}
$$

where the stress tensor $\sigma_{\alpha \beta}$ is the (negative) momentum flux tensor and the momentum source corresponds to gravitational forces with gravitational acceleration $g$. The $\mathrm{z}$ axis is oriented along the vertical direction, opposite to the gravitational field. 
Dissipation in the system is related to entropy production. The balance of the entropy density $s$ reads

$$
\frac{\partial s}{\partial t}+\nabla \cdot \mathbf{J}_{s}=\theta
$$

where $\mathbf{J}_{s}$ is the entropy flux and $\theta \geq 0$ is the local rate of entropy production per unit volume. Since energy is conserved, the energy density $u$ obeys

$$
\frac{\partial u}{\partial t}+\nabla \cdot \mathbf{J}_{u}=0
$$

where $\mathbf{J}_{u}$ denotes the energy flux. The balance of the free energy density $f=u-T s$ therefore reads

$$
\frac{\partial f}{\partial t}+\nabla \cdot\left(\mathbf{J}_{u}-T \mathbf{J}_{s}\right)=-T \theta
$$

For an isothermal system (which we consider here) with free energy density $f\left(n_{a}, n_{b}, \mathbf{v}, z\right)=$ $(1 / 2) \rho \mathbf{v}^{2}+\rho g z+f_{0}\left(n_{a}, n_{b}\right)$, the total entropy production rate $\dot{S}=\int d^{3} r \theta$ is given by

$$
\begin{aligned}
T \dot{S} & =-\int d^{3} r\left(\frac{\partial}{\partial t}\left[\frac{1}{2}\left(\rho \mathbf{v}^{2}\right)+\rho g z\right]+\sum_{i} \frac{\partial n_{i}}{\partial t} \mu_{i}\right)+\int_{\partial \Omega} d A \mathbf{n} \cdot\left(T \mathbf{J}_{s}-\mathbf{J}_{u}\right) \\
& =\int d^{3} r\left(\sigma_{\alpha \beta}^{d} u_{\alpha \beta}-\mathbf{j} \cdot \nabla \bar{\mu}\right)
\end{aligned}
$$

where $\mu_{i}=\partial f_{0} / \partial n_{i}$ are the chemical potentials of the components. The conservation laws and the Gibbs-Duhem relation $d P=n_{a} d \mu_{a}+n_{b} d \mu_{b}$ have been used to obtain the last line. Here, $\partial \Omega$ denotes the boundary surface of the volume and $\mathbf{n}$ a vector normal to the boundary pointing out of the volume. The hydrostatic pressure is denoted $P$.

The conjugate thermodynamic fluxes and forces are thus the pairs $\sigma_{\alpha \beta}^{d}, u_{\alpha \beta}$ and $\mathbf{j},-\nabla \bar{\mu}$, where $u_{\alpha \beta}=\left(\partial_{\alpha} v_{\beta}+\partial_{\beta} v_{\alpha}\right) / 2$ is the tensor of velocity gradients and the dissipative part of the stress tensor is given by $\sigma_{\alpha \beta}^{d}=\sigma_{\alpha \beta}-P \delta_{\alpha \beta}-\rho v_{\alpha} v_{\beta}$. The relevant chemical potential is the difference $\bar{\mu}=\mu_{b} / m_{b}-\mu_{a} / m_{a}$. Expressions for $\mu_{a}$ and $\mu_{b}$ in a simple model are given in Appendix A.

To linear order, the dissipative fluxes $\sigma_{\alpha \beta}^{d}, j_{\alpha}$ depend on the thermodynamic forces $u_{\alpha \beta}$, $\partial_{\alpha} \bar{\mu}$ as

$$
\begin{aligned}
j_{\alpha} & =-\gamma \partial_{\alpha} \bar{\mu} \\
\sigma_{\alpha \beta}^{d} & =2 \eta\left(u_{\alpha \beta}-\frac{1}{3} u_{\gamma \gamma} \delta_{\alpha \beta}\right)+\bar{\eta} u_{\gamma \gamma} \delta_{\alpha \beta}
\end{aligned}
$$

Here, the viscosities $\eta, \bar{\eta}$ and the dissipative coefficient $\gamma$ have been introduced. 
Hydrodynamic equations. Using Eq. (9), the solute current can be expressed as

$$
\mathbf{J}_{b}=-D \nabla n_{b}-\tilde{\gamma} \nabla P+\mathbf{v} n_{b}
$$

Here, $\quad D=\left(\gamma / m_{b}\right) \partial \bar{\mu} /\left.\partial n_{b}\right|_{P}$ is the diffusion coefficient of the solute and $\tilde{\gamma}=$ $\left(\gamma / m_{b}\right) \partial \bar{\mu} /\left.\partial P\right|_{n_{b}} \simeq\left(\gamma / m_{b}\right)\left(v_{a} / m_{a}-v_{b} / m_{b}\right)$ describes the effects of pressure gradients on solute molecules. In the limit of small solute concentration $n_{b} v_{b} \ll 1$, the dissipative coefficient scales as $\gamma \simeq \mu m_{b}^{2} n_{b}$, where $\mu$ is a mobility of solvent molecules. This scaling implies that solvent molecules contribute independently to dissipation, no interactions occur. In this limit, Eq. (11) becomes a drift-diffusion current for independent solute molecules, see Appendix B.

From the force balance (4) and the relation (10) follows the hydrodynamic equation of the barycentric flow field

$$
\eta \Delta \mathbf{v}+\left(\bar{\eta}-\frac{\eta}{3}\right) \nabla(\nabla \cdot \mathbf{v})=-\nabla P+\rho g \hat{\mathbf{e}}_{z}
$$

where $\hat{\mathbf{e}}_{z}$ is a unit vector in $z$-direction and inertial forces have been neglected. Incompressibility of the fluid implies that the molecular volumes $v_{a}$ and $v_{b}$ are constant parameters. Since the density $\rho=(1-\phi) m_{a} / v_{a}+\phi m_{b} / v_{b}$ depends on solute volume fraction $\phi$, the divergence

$$
\nabla \cdot \mathbf{v}=-\frac{\Delta \rho}{\rho}\left(\frac{\partial}{\partial t}+\mathbf{v} \cdot \nabla\right) \phi
$$

does not vanish. Here, $\Delta \rho=m_{b} / v_{b}-m_{a} / v_{a}$ is the density difference of pure solute and solvent. In the incompressible system, the pressure profile $P$ plays the role of a Lagrange multiplier function that is determined such that the corresponding flow satisfies the incompressibility condition (13).

\section{Thermodynamic equilibrium.}

The two-component fluid settles and eventually reaches an equilibrium state. At equilibrium, both the flow velocity $\mathbf{v}$ and current $\mathbf{J}_{b}$ vanish. The hydrostatic pressure obeys $\partial_{z} P=-g \rho(z)$. The solute height profile $n_{b}(z)$ at equilibrium satisfies the relation

$$
\bar{\mu}\left(n_{b}(z), P(z)\right)=\text { const. }
$$

and is independent on the dissipative coefficients. In the limit of small solute concentration $n_{b} v_{b} \ll 1$, the solute height profile becomes a barometric distribution $n_{b}(z)=\bar{n} e^{-z / \ell}$ with characteristic length $\ell=k_{B} T /\left(\Delta \rho g v_{b}\right)$, see Appendix B. 


\section{STEADY STATE GRADIENTS}

We now consider a simple geometry to discuss the main features of steady flows and concentration gradients which can be maintained stationary over long times in a two-component fluid but are intrinsically nonequilibrium states. A fluid filled channel of height $e$ along the $x$-axis, and infinite extension in $y$ direction, is connected at both ends to reservoirs with different solute concentrations $n_{b}$ or volume fractions $\phi=n_{b} v_{b}$, see Fig 1 . The left reservoir is filled with fluid of solute volume fraction $\phi_{1}$ up to a height $h_{1}$, the right reservoir up to a height $h_{2}$ at volume fraction $\phi_{2}$. We consider well-stirred reservoirs such that the solute volume fraction $\phi$ is uniform in each reservoir. Under such circumstances, $\phi$ is also constant in time for short enough times as discussed below.

For these conditions, the mass densities in the reservoirs are $\rho_{1,2}=\left(1-\phi_{1,2}\right) m_{a} / v_{a}+$ $\phi_{1,2} m_{b} / v_{b}$. The pressures in the reservoirs are given by $P_{1}=\rho_{1} g\left(h_{1}-z\right)+P_{0}$ and $P_{2}=$ $\rho_{2} g\left(h_{2}-z\right)+P_{0}$, where $P_{0}$ denotes the outside pressure. For $h_{1,2} \gg e$, the pressure gradient

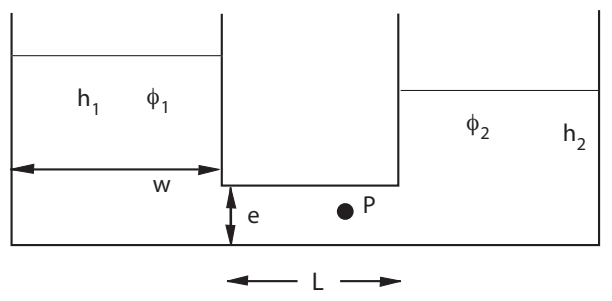

FIG. 1: Fluid channel of height $e$ and length $L$ between two reservoirs of width $w$, filled with fluid of volume fractions $\phi_{1}$ and $\phi_{2}$ and heights $h_{1}$ and $h_{2}$. In such a setting, gradients of pressure or concentration can be maintained over long times. Such gradients can drive the motion of an immersed colloidal particle $P$.

in the channel generated by the reservoirs is given by

$$
\partial_{x} P \simeq g\left(\rho_{2} h_{2}-\rho_{1} h_{1}\right) / L
$$

We consider in the following two complementary cases.

Case (A): concentration gradient in the absence of barycentric flows. In this case, the pressures on each side are balanced, $h_{1} \rho_{1}=h_{2} \rho_{2}$, and therefore, $\partial_{x} P=0$, and no flow exists, $\mathbf{v}=0$. In this case, after an initial relaxation process of duration $t_{r} \simeq L^{2} / D$, the concentration profile becomes a linear gradient

$$
\partial_{x} \phi=\left(\phi_{2}-\phi_{1}\right) / L
$$


The corresponding diffusion current is

$$
\left(J_{b}\right)_{x}=-D \frac{\phi_{2}-\phi_{1}}{L v_{b}}
$$

This current is maintained on time scales short compared to the equilibration time between reservoirs $t_{d} \simeq h w L /(e D)$, where $w$ and $h$ are reservoir width and height respectively. This time can be made arbitrarily long.

Case (B): flow without concentration gradient. In this case, the volume fractions and mass densities are equal in both reservoirs, $\phi_{1}=\phi_{2}$ and $\rho=\rho_{1}=\rho_{2}$. For $h_{1} \neq h_{2}$, a pressure gradient and a corresponding hydrodynamic flow exists $\mathbf{v}=\left(v_{x}, 0,0\right)$ with $\nabla \cdot \mathbf{v}=0$. The corresponding solution to Eq (12) is

$$
v_{x}(z) \simeq \frac{1}{2} \frac{g \rho\left(h_{2}-h_{1}\right)}{\eta L} z(z-e)
$$

In this situation, the solute concentration $n_{b}$ in the channel is constant. A solute current exists, $\left(J_{b}\right)_{x}=n_{b} v_{x}(z)+\tilde{\gamma} \partial_{x} P$, which consists of a convective part and a dissipative flux relative to the fluid flow. This barycentric flow and solute current are maintained during the

shortest of the times $t_{d} \simeq h w L /(e D)$ and $t_{c} \simeq w L \eta /\left(e^{3} \rho g\right)$. Note again that these times can be arbitrarily large. This linear concentration gradient also applies to good approximation for weak flows if $D / v_{x} \gg L$. For larger flow velocities, nonlinear concentration fields occur.

\section{DISSIPATIVE INTERFACIAL PROCESSES: SURFACE SLIP VELOCITIES}

A colloidal particle that is immersed in a two component fluid as described above can be set in motion as a result of nonequilibrium conditions provided by either a pressure gradient or a solute gradient. In order to determine the velocity of motion of the bead relative to the flow, and the corresponding perturbation of the flow field, the hydrodynamic equations of the fluid are solved with appropriate boundary conditions applied at the particle surface. These boundary conditions can be systematically derived by expressing surface dissipation at the fluid particle interface and writing generic Onsager relations for conjugate forces and fluxes at the interface.

We consider a solid substrate in contact with a two-component fluid. We use a coordinate system with $z$ denoting the distance from the solid surface along the normal direction. In the interface, we average all quantities over the thickness $d$ in which properties differ from 
bulk properties. This procedure is valid when $d \ll R$, where $R^{-1}$ is a local curvature of the interface. The interface dissipation rate reads (see Appendix D)

$$
T \dot{S}=\int d A\left(\frac{1}{2} \sigma_{i z}^{s} v_{i}^{s}-j_{i}^{s} \nabla_{i} \bar{\mu}^{s}+r^{s} \Delta \mu\right)
$$

Here, the indicies $i, j$ denote directions parallel to the interface. The superscripts $s$ indicate that surface fields are considered, $v_{i}^{s}=v_{i}(z=0)$ and $\sigma_{i z}^{s}=\sigma_{i z}(z=0)$. Note that we have neglected for simplicity surface viscosity and $j_{i}^{s}$ is a surface current with units of mass per length and time. To keep our discussion simple, we consider here the case where the interface equilibrates rapidly with the fluid [1], $\bar{\mu}^{s}=\bar{\mu}(z=0)$ and material exchange between interface and fluid can be negected $v_{z}(z=0)=0$ and $j_{z}(z=0)=0$.

In addition to the two conjugate fluxes and forces already discussed, we have added here the term $r^{s} \Delta \mu$ which describes an active process on the interface which can propel a swimmer. An example from biology would be a large number beating of cilia on the particle surface. The active process is driven by a chemical fuel (which in a living cell would be ATP) with chemical potential difference between fuel and product $\Delta \mu$. The conjugate flux $r^{s}$ denotes the rate of fuel consumption per unit area of the surface. The active process can only contribute to net motion generation if the surface has a vectorial asymmetry which for example determines the direction of the active stroke of a cilium along the surface. This direction is characterized by a normalized vector $p_{i}$ tangent to the surface.

The three pairs of conjugate fluxes and forces are related to linear order by

$$
\begin{aligned}
& v_{i}^{s}=\nu \sigma_{i z}^{s}-\alpha \nabla_{i} \bar{\mu}^{s}+p_{i} \zeta \Delta \mu \\
& j_{i}^{s}=-\alpha \sigma_{i z}-\gamma^{s} \nabla_{i} \bar{\mu}^{s}+p_{i} \zeta^{\prime} \Delta \mu \\
& r^{s}=\zeta p_{i} \sigma_{i z}^{s}+\zeta^{\prime} p_{i} \nabla_{i} \bar{\mu}^{s}+\Lambda \Delta \mu,
\end{aligned}
$$

where the coefficients satisfy Onsager symmetry relations. We observe that there exists in general a finite slip velocity $v_{i}^{s}$ at the surface. The coefficient $\nu$ can be characterized by the related "slip length" $b=\nu \eta$, which is the distance from the surface at which an effective no slip boundary condition applies. The coefficient $\gamma^{s}$ is related to surface diffusion and $\alpha$ is a dissipative coefficient which couples surface flows to relative fluxes between solvent and solute. The coefficients $\zeta$ and $\zeta^{\prime}$ describe the coupling of the active process on the surface to the two-component fluid. 
We now consider the case $\zeta=0$ and $\zeta^{\prime}=0$. We furthermore assume that the slip length vanishes, $\nu=0$, which implies that there is no slip in the absence of a chemical potential gradient. There remains a surface slip velocity driven by chemical potential gradients and pressure gradients, which as in the bulk can be expressed as

$$
v_{i}^{s}=-\kappa \nabla_{i} n_{b}^{s}-\kappa^{\prime} \nabla_{i} P^{s}
$$

Here $\kappa=\left.\left(\alpha / m_{b}\right)\left(\partial \bar{\mu} / \partial n_{b}\right)\right|_{z=0}$ and $\kappa^{\prime}=\left.\left(\alpha / m_{b}\right)(\partial \bar{\mu} / \partial P)\right|_{z=0}$. For a specific interface model,

the coefficient $\kappa$ has been expressed as $\kappa \simeq k_{B} T \lambda^{2} / \eta[1]$, where the length scale $\lambda$ is related to the range of the potential describing interactions between solute molecules and the particle surface and is of the order of the interface thickness $d$.

\section{COLLOIDAL TRANSPORT}

We now consider a spherical colloidal particle of radius $a$ which is subject to the solute concentration gradient or pressure gradient. We are interested in the particle velocity $v_{p}=$ $v_{x}+\Delta v$ in $x$-direction in the laboratory frame where $\Delta v$ is the velocity difference between particle and flow. These velocities can be determined by solving the Stokes equation (12) with the appropriate slip boundary conditions on the particle surface.

In the following, we consider the case where the particle is far from any walls which allows us to ignore the effects of boundaries of the channel in which the particle is placed. We ignore sedimentation of the particle in the gravitational field in $z$-direction. We discuss two cases (A) and (B) described above.

(A) Concentration gradient in the absence of barycentric flows. In the presence of a constant concentration gradient $\partial_{x} n_{b}=\left(\phi_{1}-\phi_{2}\right) /\left(L v_{b}\right)$, a slip velocity at the particle surface is generated. We consider the case of zero slip length $b=0$ and no active process $\Delta \mu=0$. In spherical coordinates of the particle $r, \theta$, where $\theta$ measures the angle with respect to the gradient direction along the $x$-axis, and $r$ is the radial distance from the particle center, the slip velocity $\mathbf{v}^{s}=v_{s}(\theta) \mathbf{e}_{\theta}$, is given by

$$
v_{s}(\theta)=-\kappa \sin (\theta) \partial_{x} n_{b}
$$

where $\mathbf{e}_{\theta}$ is a unit vector in $\theta$ direction tangential to the sphere. Using the boundary conditions $v_{\theta}(r=a, \theta)=v_{s}(\theta)$ and $v_{r}(r=a, \theta)=0$, the flow field around a spherical 
particle is given by (see Appendix E)

$$
\begin{aligned}
& v_{r}(r, \theta)=-\Delta v\left(1-\frac{a^{3}}{r^{3}}\right) \cos (\theta) \\
& v_{\theta}(r, \theta)=\frac{\Delta v}{2}\left(2+\frac{a^{3}}{r^{3}}\right) \sin (\theta)
\end{aligned}
$$

where we have used for simplicity the condition $\nabla \cdot \mathbf{v}=0$ which is according to Eq. (13) satisfied if $v_{a} / m_{a}=v_{b} / m_{b}$. The relative velocity between particle and fluid far from the particle is

$$
\Delta v=(2 \kappa / 3) \partial_{x} n_{b}
$$

There is no body force acting on the particle, $f^{p}=0$, see Appendices $\mathrm{E}$ and $\mathrm{F}$. The flow field perturbation decays as $\sim 1 / r^{3}$ for increasing $r$ and thus faster than a Stokeslet which decays as $\sim 1 / r$ and is the signature of a net force. The decay $\sim 1 / r^{3}$ corresponds to a source doublet and implies that a force quadrupole is exerted by the particle on the fluid. Here, no force dipole exists. In more general situations, a force dipole also exists, which correspond to a decay of the velocity $\sim 1 / r^{2}$, see Appendix E. Note that a force dipole does not contribute to propulsion but dominates the far field. Note also, that there are no forces due to osmotic pressures acting on a particle in a concentration gradient, see Appendix C.

(B) Flows in the absence of concentration gradients: In the presence of a pressure gradient $\partial_{x} P$, a parabolic flow profile (18) is generated. If we can ignore the effects of walls, and if no surface slip occurs, the relative velocity of the particle with respect to the flow $\Delta v_{1}=v_{p}-v_{x}$ can be determined by Faxens law as [15] (see Appendix E)

$$
\Delta v_{1}=\frac{3 a^{2} \partial_{x} P}{4 \eta}
$$

Surface slip induces an additional component $\Delta v_{2}$ to the relative motion between fluid and particle. For vanishing slip length $b=0$, the only contribution to the slip is $v_{i}^{s}=-\kappa^{\prime} \nabla_{i} P^{s}$. The boundary conditions in a coordinate frame co-moving with the particle are $v_{r}(\theta, r=$ $a)=0$ and $v_{\theta}(\theta, r=a)=-\kappa^{\prime} \sin (\theta) \partial_{x} P$. The flow field perturbation generated by surface slip is given by Eq. (25) with $\Delta v=\Delta v_{2}=\left(2 \kappa^{\prime} / 3\right) \partial_{x} P$.

Since $\kappa^{\prime}$ expresses the momentum transfer to the particle within the interfacial layer of thickness $d$, one expects $\kappa^{\prime} \simeq-\bar{d}^{2} / \eta$, where the length $\bar{d}$ is of order $d$. By superimposing the flow field (18) with the perturbation by the particle $\Delta v_{1}$ and $\Delta v_{2}$, the overall particle 
velocity is

$$
v_{p} \simeq-\left[\frac{e^{2}}{8 \eta}-\frac{a^{2}}{12 \eta}+\frac{2 \bar{d}^{2}}{3 \eta}\right] \frac{g\left(\rho_{2} h_{2}-\rho_{1} h_{1}\right)}{L}
$$

which is entirely driven by the pressure gradient. No net body force $f^{p}$ acts on the particle, see Appendix F. Note that the convective term is large compared to $\Delta v_{1}$ which is in turn large compared to $\Delta v_{2}$. The existence of $\Delta v_{1}$ is yet important since it can lead to separation of particles according to their size.

\section{SELF-PROPULSION}

Propulsion by self-generated concentration gradients. A chemical reaction catalyzed on the particle surface can generate local concentration gradients which propel the particle [4, 5]. To describe situations where the particle catalyzes a reaction involving two solvent species, at least a three-component fluid description is required. However, the basic physics of self-propulsion can be captured by our two-component fluid if we assume that a surface reaction transforms molecules of type $a$ into molecules of type $b$. In this case, we consider two components with equal molecular masses $m_{a}=m_{b}$ to satisfy mass conservation. The molecular volumes $v_{a}$ and $v_{b}$ can in general differ. A local reaction rate $S$ per unit area at which $a$ molecules are transformed in $b$ molecules on the particle surface implies the boundary conditions for the molecular fluxes normal to the particle surface

$$
\left(J_{b}\right)_{z}(z=0)=-\left(J_{a}\right)_{z}(z=0)=S
$$

in the reference frame where the particle is at rest. The corresponding boundary condition for the center of mass velocity is $v_{z}(z=0)=0$.

The nonlinear convective term in the flux Eq. (11), couples the concentration and flow fields. In the limit of small Peclet number $\mathrm{Pe}=\Delta v a / D$, we can neglect this convective nonlinearity. The stationary concentration field is then solution to the diffusion equation $\nabla^{2} \phi=0[4,[5]$. For the simple choice of an asymmetrically distributed reaction rate $S(\theta)=$ $S_{0} \cos (\theta)$, the solution for the boundary conditions specified above is given by

$$
n_{b}(r, \theta)=\frac{S_{0} a^{3}}{2 D r^{2}} \cos (\theta)+n_{b}^{\infty}
$$

where $n_{b}^{\infty}$ denotes the concentration far from the particle. This concentration field induces 
according to Eq. (23) the surface slip velocity

$$
v_{s}(\theta)=\frac{\kappa S_{0}}{2 D} \sin (\theta)
$$

which is independent of particle radius. The corresponding hydrodynamic flow field for the case $v_{a}=v_{b}$ for which $\nabla \cdot \mathbf{v}=0$ is given by Eq. (25) with

$$
\Delta v=\frac{\kappa S_{0}}{3 D}
$$

The pressure $P$ is constant and there is no net body force $f^{p}$ acting on the particle.

For finite Peclet number, there exist no simple solutions to this nonlinear problem since the convection velocity in Eq. (11) is given by the hydrodynamic flow field described by Eq. (12). The latter in turn is coupled to the concentration field $n_{b}$ via the surface slip driven by the chemical reaction. Here, we focus on the scaling behavior of the propulsion velocity for large Pe. The hydrodynamic flow relative to the particle is of order $\Delta v$. This defines a length scale $\ell \simeq(D a / \Delta v)^{1 / 2} \ll a$ which characterizes the thickness of the boundary layer near the particle in which the concentration field is different from $n_{b}^{\infty}$ by a variation of order $\Delta n_{b} \simeq \ell S_{0} / D$. The resulting slip velocity is of the order $\Delta v \simeq \kappa \Delta n_{b} / a$. By combining these expressions, we obtain

$$
\Delta v \simeq \frac{\kappa^{2 / 3} S_{0}^{2 / 3}}{D^{1 / 3} a^{1 / 3}}
$$

The velocity thus increases for large Pe less than linearly and becomes dependent on the particle size $a$. For $\mathrm{Pe}=1$, the relations (32) and (33) match except for a dimensionless prefactor.

Propulsion by active surface processes. Many microorganisms swim by using a large number of cilia attached to the surface which generate periodic beating movements that are driven by molecular motors that consume a chemical fuel [7]. In a coarse-grained picture where the cilia are active elements within the interface between swimmer and fluid, the motion of the cilia effectively generates a surface slip velocity of the flow. In our generic description this surface slip is captured by the term $v_{i}^{s} \simeq p_{i} \zeta \Delta \mu$ in Eq. (20). Here, the tangent vector $p_{i}$ describes the direction along which the cilium generates a flow and $\zeta$ is a coupling coefficient between the free energy $\Delta \mu$ driving the motors and the surface flow. In this scenario, the slip pattern on the surface of the swimmer is determined by the ciliar beat direction and strength. 
For given surface slip pattern and corresponding hydrodynamic flows with $\nabla \cdot \mathbf{v}=0$, the velocity of the swimmer can be determined without explicit calculation of the flow field. For a spherical particle it is given by [16]

$$
\Delta \mathbf{v}=\frac{1}{4 \pi a^{2}} \int d A \mathbf{v}^{s}
$$

Similarly, the rotation rate and axis (which can exist for a surface slip velocity field lacking axial symmetry) is described by [16]

$$
\Omega=\frac{3}{8 \pi a^{3}} \int d A \mathbf{n} \times \mathbf{v}^{s}
$$

where $\mathbf{n}$ is a unit vector normal to the surface and $\boldsymbol{\Omega}$ points in the direction of the rotation axis. Again, this translational and rotational motion occurs relative to the fluid without a net body force and torque acting on the swimmer. For the simple example with $p_{i}$ a unit tangent vector in $\theta$ direction and $\zeta=\zeta_{0} \sin (\theta)$, we have $\Delta v=(2 / 3) \zeta_{0} \Delta \mu$ and again the flow field perturbation of Eq. (25).

\section{DISCUSSION}

We have presented a generic description of colloidal transport to clarify the force balances involved and the role of interfacial slip. The simplest form of transport occurs if relative external body forces $f^{p}$ are applied to a particle. External body forces, such as those due to gravitation, correspond to source terms in the momentum balance (4). As a result, the particle moves at a speed $\Delta v$ relative to the fluid and experiences Stokes friction $f^{p}=$ $6 \pi \eta a \Delta v$. The corresponding perturbation of the fluid flow at large distances is given by a Stokeslet which decays as $1 / r$.

In the absence of external fields, a colloidal particle in a fluid is force free, $f^{p}=0$, even if concentration or pressure gradients exist. In this case a colloidal particle nevertheless moves relative to the fluid at a velocity $\Delta v$ if there is a slip of the flow at the surface of the particle. Such slip is generated by surface dissipative phenomena [1]. We have shown that Onsager relations on the solid surface determine the boundary conditions for the hydrodynamic equations in the bulk. This description can account for a variety of phenomena, including a slip length, slip induced by concentration or pressure gradients as well as slip due to active processes on the particle surface, within a unified framework. 
Our generic description can be generalized to electric fields, which requires to replace chemical potentials by electrochemical potentials and to include electrostatics in the free energy density. Effects in the presence of electric fields include electrophoresis of charged particles. Since a charged particle is screened beyond an electric double layer of counter ions, it is effectively neutral. Electrophoresis is thus another example where no force acts on a particle (including the layer of counter ions) and electrophoretic motion results thus from surface slip [1, 17].

Our arguments are relevant for mechanisms of self-propulsion of colloidal particles. Self propulsion implies that motion occurs in the absence of externally applied forces. This is possible if a particle self-generates a surface slip velocity by active processes on or near the particle surface. These include the action of cilia and flagella in the case of swimming microorganisms [7] or the generation of a concentration gradient by surface chemical reactions [6]. The main effect of the surface slip is to generate a relative motion between particle and fluid. In the general case, this relative motion is associated with a flow field perturbation that decays as $\sim 1 / r^{2}$, corresponding to a force dipole exerted by the particle on the fluid which however does not contribute to propulsion. If no force dipole exists, such as in the case described by Eq. (25), the flow perturbation decays as $\sim 1 / r^{3}$. This flow perturbation corresponds to a force quadrupole acting on the fluid. In all cases there is no net external force acting on the particle.

In a recent publication it has been claimed that self-generated concentration gradients generate a force on a particle by osmotic pressure gradients which is balanced by Stokes friction [13]. Clearly, no force is exerted on a particle in a concentration field by osmotic pressure, see Appendix C. The scenario proposed in [13] violates momentum conservation, see Appendix F. The work of Ref. [13] furthermore ignores the effect of hydrodynamic flow perturbations on the concentration gradients, when those cannot be neglected at finite Peclet numbers. Our generic description of colloidal transport can serve to clarify these and related points in a systematic way.

We thank Ramin Golestanian for stimulating discussions. F.J. thanks Friederike Schmidt, Holger Stark and Andrej Vilfan for similarly stimulating discussions on the hydrodynamic flow perturbations generated by colloidal particles and swimmers. 


\section{APPENDIX A: ENTROPY OF MIXING AND CHEMICAL POTENTIALS}

The properties of the chemical potentials can be discussed using a simple model for a two-component fluid with a free energy in the rest frame $F_{0}\left(N_{a}, N_{b}, V\right)=V f_{0}\left(N_{a} / V, N_{b} / V\right)$, where $V$ denotes volume, with

$$
f_{0}\left(n_{a}, n_{b}\right)=k_{B} T\left(n_{a} \ln \frac{n_{a} v_{a}}{n_{a} v_{a}+n_{b} v_{b}}+n_{b} \ln \frac{n_{b} v_{b}}{n_{a} v_{a}+n_{b} v_{b}}\right)+\frac{\chi}{2}\left(n_{a} v_{a}+n_{b} v_{b}-1\right)^{2}
$$

Here, the first term describes the entropy of mixing of two components with molecular volumes $v_{a}$ and $v_{b}$, the second term describes the compressibility of the fluid by the coefficient $\chi$. Interactions between the two components are neglected. The chemical potentials $\mu_{i}=$ $\partial f_{0} / \partial n_{i}$ are given by

$$
\begin{aligned}
& \mu_{a}\left(n_{a}, n_{b}\right)=k_{B} T\left(\ln \frac{n_{a} v_{a}}{n_{a} v_{a}+n_{b} v_{b}}+\frac{n_{b}\left(v_{b}-v_{a}\right)}{n_{a} v_{a}+n_{b} v_{b}}\right)+v_{a} \chi\left(n_{a} v_{a}+n_{b} v_{b}-1\right) \\
& \mu_{b}\left(n_{a}, n_{b}\right)=k_{B} T\left(\ln \frac{n_{b} v_{b}}{n_{a} v_{a}+n_{b} v_{b}}+\frac{n_{a}\left(v_{a}-v_{b}\right)}{n_{a} v_{a}+n_{b} v_{b}}\right)+v_{b} \chi\left(n_{a} v_{a}+n_{b} v_{b}-1\right)
\end{aligned}
$$

The pressure $P=-\left.\left(\partial F_{0} / \partial V\right)\right|_{N_{a}, N_{b}}=-f_{0}+\mu_{a} n_{a}+\mu_{b} n_{b}$ is

$$
P\left(n_{a}, n_{b}\right)=\frac{\chi}{2}\left(\left(n_{a} v_{a}+n_{b} v_{b}\right)^{2}-1\right)
$$

Using the pressure, the solvent density $n_{a}$ can be eliminated. In the incompressible limit of large $\chi, n_{a} v_{a}+n_{b} v_{b}=1$ and we obtain

$$
\begin{aligned}
& \mu_{a}\left(n_{b}, P\right) \simeq k_{B} T\left(\ln \left(1-n_{b} v_{b}\right)+n_{b}\left(v_{b}-v_{a}\right)\right)+P v_{a} \\
& \mu_{b}\left(n_{b}, P\right) \simeq k_{B} T\left(\ln \left(n_{b} v_{b}\right)+n_{a}\left(v_{a}-v_{b}\right)\right)+P v_{b}
\end{aligned}
$$

\section{APPENDIX B: LIMIT OF SMALL SOLUTE CONCENTRATION}

The solute flux is driven by gradients of the chemical potential difference $\bar{\mu}=\mu_{b} / m_{b}-$ $\mu_{a} / m_{a}$

$$
\mathbf{J}_{b}=-\frac{\gamma}{m_{b}} \nabla \bar{\mu}+n_{b} \mathbf{v}
$$

In the limit $n_{b} v_{b} \ll 1$ of small solute concentration,

$$
\bar{\mu}\left(n_{b}, P\right) \simeq \frac{k_{B} T}{m_{b}} \ln \left(n_{b} v_{b}\right)+\left(\frac{v_{b}}{m_{b}}-\frac{v_{a}}{m_{a}}\right) P
$$


For small $n_{b}$, solute particles become independent of each other and dissipation takes place independently for each solute particle. Therefore, $\gamma \simeq \xi m_{b}^{2} n_{b}$, where $\xi$ is a mobility per solute molecule. We thus find

$$
\mathbf{J}_{b} \simeq-D \nabla n_{b}-\bar{\gamma} n_{b} \nabla P+n_{b} \mathbf{v}
$$

where

$$
\begin{aligned}
\bar{\gamma} & \simeq \xi m_{b}\left(v_{b} / m_{b}-v_{a} / m_{a}\right) \\
D & \simeq \xi k_{B} T
\end{aligned}
$$

In the limit of small $n_{b} v_{b}$, the pressure gradient is approximately constant $\partial_{z} P=-\rho g \simeq$ $-g m_{a} / v_{a}$. For $\mathbf{v}=0$, the height profile is

$$
n_{b}=\bar{n} e^{-z / \ell}
$$

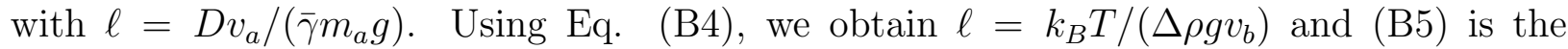
barometric height distribution.

\section{APPENDIX C: OSMOTIC PRESSURE}

Osmotic pressures are a consequence of a semipermeable interface which separates the fluid in two compartments (1) and (2). The solvent passes this interface, which is impermeable to the solute. As a consequence, across the interface the chemical potential of the solvent is balanced, $\mu_{a}^{(1)}=\mu_{a}^{(2)}$. However, the solute chemical potentials do not balance $\mu_{b}^{(1)} \neq \mu_{b}^{(2)}$.

The solvent chemical potential is according to Eq. (A5) in the limit of small $n_{b} v_{b}$ given by $\mu_{a} \simeq-k_{B} T n_{b} v_{a}+P v_{a}$. The balance of solvent chemical potentials implies the existence of an osmotic pressure difference across the semipermeable membrane

$$
P^{(2)}-P^{(1)}=k_{B} T\left(n_{b}^{(2)}-n_{b}^{(1)}\right)
$$

Note that the hydrostatic pressure difference appears only after the balance of the chemical potential of the solvent is reached and that the corresponding momentum source is provided by the membrane. 


\section{APPENDIX D: FORCE BALANCE AND DISSIPATION AT AN INTERFACE}

We consider dissipation and force balances in an interfacial region of thickness $d$ between two phases in which material properties differ from those in the two bulk phases. The coordinate normal to the interface is denoted $z$. A relative slip velocity can occur at an interface. The local center of mass velocity tangential to the interface at $z= \pm d / 2$ is denoted $v_{i}^{ \pm}$, respectively. Dissipation due to interfacial slip

$$
v_{i}^{s}=v_{i}^{+}-v_{i}^{-}
$$

can be expressed as

$$
T \dot{S} \simeq \int d A \int_{-d / 2}^{d / 2} d z \frac{\partial_{z} v_{i}}{2} \sigma_{i z} \simeq \frac{1}{2} \int d A \sigma_{i z}^{s} v_{i}^{s}
$$

The interfacial shear stress $\sigma_{i z}^{s}=\epsilon \sigma_{i z}^{+}+(1-\epsilon) \sigma_{i z}^{-}$is a weighted average of $\sigma_{i z}^{ \pm}$. The value of $0<\epsilon<1$ depends on the internal structure of the interface. The conjugate thermodynamic variables are thus $v_{i}^{s}$ and $\sigma_{i z}^{s}$. The corresponding Onsager relation reads

$$
v_{i}^{+}-v_{i}^{-}=\nu\left(\epsilon \sigma_{i z}^{+}+(1-\epsilon) \sigma_{i z}^{-}\right)
$$

where $\nu$ is the corresponding dissipative coefficient.

The force balance in the interfacial region $\partial_{\beta} \sigma_{\alpha \beta}=0$ implies

$$
\int_{-d / 2}^{d / 2} d z\left(\partial_{z} \sigma_{i z}+\partial_{j} \sigma_{i j}\right)=0
$$

This implies the interfacial force balance

$$
\sigma_{i z}^{+}-\sigma_{i z}^{-}=-\partial_{i} \Sigma
$$

where the interfacial tension (for isotropic stresses in the tangent plane) is

$$
\Sigma=\frac{1}{2} \int_{-d / 2}^{d / 2} d z \sigma_{k k}
$$

In the absence of interfacial tension gradients $\partial_{i} \Sigma$, the shear stress is continuous across the interface $\sigma_{i z}^{s}=\sigma_{i z}^{+}=\sigma_{i z}^{-}$. and the slip velocity is simply given by $v_{i}^{s}=\nu \sigma_{i z}^{+}$as in Eq. (20). 


\section{APPENDIX E: HYDRODYNAMIC FLOW FIELDS WITH AXIAL SYMMETRY}

Solutions to the Stokes Eq. (12) for axisymmetric incompressible flows with $\nabla \cdot \mathbf{v}=0$ can be expressed using the stream function $\psi$ [15]. In spherical coordinates, the velocity field is related to the stream function $\psi(r, \theta)$ by

$$
\begin{aligned}
& v_{r}=-\frac{1}{r^{2} \sin \theta} \frac{\partial \psi}{\partial \theta} \\
& v_{\theta}=\frac{1}{r \sin \theta} \frac{\partial \psi}{\partial r}
\end{aligned}
$$

The stream function satisfies the differential equation $E^{4} \psi=0$, where

$$
E^{2} \psi=\left(\frac{\partial^{2}}{\partial r^{2}}+\frac{\sin \theta}{r^{2}} \frac{\partial}{\partial \theta} \frac{1}{\sin \theta} \frac{\partial}{\partial \theta}\right) \psi
$$

Simple solutions are given by

$$
\psi=\sin ^{2} \theta\left(A_{1} r^{4}+A_{2} r^{2}+A_{3} r+\frac{A_{4}}{r}\right)
$$

where $A_{1}, \ldots, A_{4}$ are constant parameters determined by boundary conditions. The corresponding pressure field is given by $P=-\eta \cos \theta\left(20 A_{1} r+2 A_{3} / r^{2}\right)+P^{\infty}$, where $P^{\infty}$ is the pressure far from the particle. The body force acting on the particle which is balanced by forces exerted by the hydrodynamic flow is $f^{p}=-8 \pi \eta A_{3}[15]$.

In case $(\mathrm{A})$, we determine a solution of the form given by Eq. (E4) with $v_{\theta}(r=a)=v_{s}(\theta)$ and $v_{r}(r=a)=0$ in the reference frame moving with the sphere. For large $r$, we require motion at constant velocity $\Delta v$ in negative $x$-direction, $v_{r} \simeq-\Delta v \cos (\theta)$ and $v_{\theta} \simeq \Delta v \sin \theta$. From the latter conditions, it follows that $A_{1}=0$, and $2 A_{2}=-\Delta v$. Because there is no external force acting on the particle, $f^{p}=0$ and thus $A_{3}=0$. The boundary conditions on the particle surface imply $A_{4}=-A_{2} a^{3}$ and $3 A_{2}=-\left(\kappa / v_{b}\right) \partial_{x} \phi$. The corresponding flow is given by Eq. (25). The perturbation of the flow velocity decaying as $\sim 1 / r^{3}$ corresponds to a source doublet [15, 18] and implies that a force quadrupole is exerted by the particle on the fluid.

In case (B), we superimpose the flow (18) driven by an applied pressure gradient with the velocity $\Delta v_{1}$ due to the perturbation of the Poiseuille flow by the sphere and the relative velocity $\Delta v_{2}$ due to interfacial slip. The velocity $\Delta v_{1}$ can be estimated from Faxens theorem which expresses the force on the particle as

$$
f_{x}^{p}=6 \pi \eta a\left(v_{p}-v_{x}^{0}\right)+\pi a^{3} \nabla^{2} v_{x}^{0}
$$


where $v_{x}^{0}$ denotes the unperturbed parabolic flow field. No force acts on the particle, $f_{x}^{p}=0$, which determines the relative velocity $\Delta v_{1}=v_{p}-v_{x}^{0}$. This flow is superimposed with a flow driven by slip boundary conditions (25) as described for case (A).

If a net body force $f^{p}$ acts on the particle with no slip, the boundary conditions are $v_{r}(r=a)=0$ and $v_{\theta}(r=a)=0$ which require $3 A_{4}=A_{3} a^{2}$ and $A_{2}=-(2 / 3) A_{3}$. From the asymptotic behavior, it follows that $A_{1}=0$ and $2 A_{2}=-\Delta v$. This implies $f^{p}=6 \pi \eta a \Delta v$ and the flow field is given by

$$
\begin{aligned}
& v_{r}(r, \theta)=-\Delta v\left(1-\frac{3}{2} \frac{a}{r}+\frac{a^{3}}{2 r^{3}}\right) \cos (\theta) \\
& v_{\theta}(r, \theta)=\Delta v\left(1-\frac{3}{4} \frac{a}{r}-\frac{a^{3}}{4 r^{3}}\right) \sin (\theta)
\end{aligned}
$$

In addition to the force monopole this flow contains again a contribution from a source doublet implying a force quadrupole.

The equation $E^{4} \psi=0$ for the flow also has the solution

$$
\psi=-\cos \theta \sin ^{2} \theta\left(B_{1}+B_{2} / r^{2}\right)
$$

The corresponding flow field is $v_{r}=\left(3 \cos ^{2} \theta-1\right)\left(B_{1} / r^{2}+B_{2} / r^{4}\right)$ and $v_{\theta}=B_{2} \sin (2 \theta) /\left(2 r^{4}\right)$ [9]. The condition $v_{r}(r=a)=0$ that the radial flow vanishes on the particle surface imposes $B_{2}=-B_{1} a^{2}$. The decay of the radial component proportional to $\sim 1 / r^{2}$ corresponds to a stokes doublet which implies the action of a force dipole on the fluid [18]. This force dipole dominates in the far field over the force quadarupole. For an arbitrary distribution of surface slip $v_{\theta}(r=a)=v_{s}(\theta)$, a force dipole exists in general. The force dipole vanishes by symmetry if the surface slip is a symmetric function $v_{s}(\theta)=v_{s}(\pi-\theta)$ such as is the case for $v_{s} \sim \sin (\theta)$ described by Eq. (25). Note that the flow perturbation corresponding to a force dipole does not contribute to propulsion by symmetry.

APPENDIX F: GENERAL CONSIDERATIONS CONCERNING THE EXISTENCE OR NON-EXISTENCE OF A STOKESLET IN A VELOCITY FIELD CARRYING A PARTICLE

Consider a particle of any shape, including arbitrary topological genus, immersed in a multi-component fluid flowing in a container of complex geometry possibly of non trivial topology. The fluid is submitted to an external force of density $g_{\alpha}^{f \text {,ext }}$ and the particle to the 
force density $g_{\alpha}^{p \text {,ext }}$. The momentum flux in the fluid is characterized by a stress tensor $\sigma_{\alpha \beta}$. The total force acting on the particle reads:

$$
f_{\alpha}^{\mathrm{tot}}=\int_{V_{p}} g_{\alpha}^{p, \text { ext }} d V+\int_{S_{p}} \sigma_{\alpha \beta} d A_{\beta}
$$

Here, the integration volume $V_{p}$ and surface $S_{p}$ refer to the particle and the surface elements $d A_{\beta}$ are oriented to point outward from the particle. The particle dynamics is $m \ddot{x}_{\alpha}=f_{\alpha}^{\text {tot }}$, where $x_{\alpha}$ is the particle position and $m$ is the particle mass. In the Stokes limit where inertial terms can be neglected or in stationary conditions $f_{\alpha}^{\text {tot }}=0$, which implies that in inertia free regimes the total force acting on a particle in a fluid vanishes. Momentum conservation in the fluid implies

$$
g_{\alpha}^{f, \text { ext }}+\partial_{\beta} \sigma_{\alpha \beta}=0
$$

where inertial forces have again been neglected. In integral form, this can be expressed as

$$
\int_{S_{p}} \sigma_{\alpha \beta} d A_{\beta}+\int_{S} \sigma_{\alpha \beta} d A_{\beta}=-\int_{V-V_{p}} g_{\alpha}^{f, \text { ext }} d V
$$

Here the integration is over the particle surface $S_{p}$ and an arbitrary surface $S$ enclosing a volume $V$ which includes the particle in the fluid. The surface elements are oriented to point to the outside of the fluid volume $V-V_{p}$ between the surfaces $S_{p}$ and $S$. The (vanishing) total force now reads

$$
f_{\alpha}^{\text {tot }}=\int_{V_{p}} g_{\alpha}^{p, \text { ext }} d V+\int_{S} \sigma_{\alpha \beta} d A_{\beta}+\int_{V-V_{p}} g_{\alpha}^{f, \text { ext }} d V
$$

or equivalently,

$$
f_{\alpha}^{\text {tot }}=\int_{V_{p}}\left(g_{\alpha}^{p, \text { ext }}-g_{\alpha}^{f, \text { ext }}\right) d V+\int_{S} \sigma_{\alpha \beta} d A_{\beta}+\int_{V} g_{\alpha}^{f, \text { ext }} d V
$$

Consider now the stress $\sigma_{i j}^{0}$ in the fluid in the absence of the particle (every other condition being kept identical). For any volume and corresponding surface we have

$$
\int_{V} g_{\alpha}^{f, \operatorname{ext}} d V=-\int_{S} \sigma_{\alpha \beta}^{0} d A_{\beta}
$$

Therefore, for any surface $S$ enclosing the particle in the fluid,

$$
f_{\alpha}^{\text {tot }}=\int_{V_{p}}\left(g_{\alpha}^{p, \text { ext }}-g_{\alpha}^{f, \text { ext }}\right) d V+\int_{S}\left(\sigma_{\alpha \beta}-\sigma_{\alpha \beta}^{0}\right) d A_{\beta}=0
$$

We thus find that the relative external body force

$$
f_{\alpha}^{p}=\int_{V_{p}}\left(g_{\alpha}^{p, \text { ext }}-g_{\alpha}^{f, \text { ext }}\right) d V
$$


which includes an Archimedian correction to the net body force, is balanced by the stresses exerted by the perturbation of the flow field on the particle

$$
f_{\alpha}^{p}=-\int_{S}\left(\sigma_{\alpha \beta}-\sigma_{\alpha \beta}^{0}\right) d A_{\beta}
$$

(for the choice $S=S_{p}$ ).

Eq. (F9) is valid for arbitrary $S$ enclosing the particle. Therefore, for colloidal particles far from the boundaries of the fluid, the total momentum flux through any closed surface is constant and equal to $f_{\alpha}^{p}$. This implies that the stress field perturbation $\sigma_{\alpha \beta}-\sigma_{\alpha \beta}^{0} \sim 1 / r^{2}$ for large $\mathrm{r}$, where $r$ is the distance from the particle center. Since $\sigma_{\alpha \beta}-\sigma_{\alpha \beta}^{0} \sim\left(\partial_{\alpha} v_{\beta}+\partial_{\beta} v_{\alpha}\right)$, the barycentric velocity perturbation scales as $|\mathbf{v}| \sim 1 / r$ which implies the existence of a Stokeslet. If the relative external body force $f_{\alpha}^{p}$ vanishes, but a relative external torque acts on the particle, a similar argument using angular moment conservation implies that in this case the stress scales as $\sim 1 / r^{3}$ and the velocity field like $\sim 1 / r^{2}$.

If no external field acts on either the particle or the fluid, there is neither external body force no torque. The far field of the hydrodynamic flow is dominated by a force dipole or Stokes doublet with stress $\sim 1 / r^{3}$ and velocity $\sim 1 / r^{2}$. If the force dipole vanishes, the stress scales as $\sim 1 / r^{4}$ and the velocity as $\sim 1 / r^{3}$. As we have illustrated in this work, the absence of external forces and torques does not mean that there is no motion between particle and fluid.

[1] J. Anderson, Annu. Rev. Fluid Mech. 21, 61 (1989).

[2] M. Leonetti, Europhys. Lett. 32, 561 (1995).

[3] P. Lammert, J. Prost, and R. Bruinsma, J. Theor. Biol. 178, 387 (1996).

[4] R. Golestanian, T. B. Liverpool, and A. Ajdari, Phys. Rev. Lett. 94, 220801 (2005).

[5] R. Golestanian, T. B. Liverpool, and A. Ajdari, New J. Phys. 9, (2007).

[6] J.R. Howse, R.A.L. Jones, A.J. Ryan, T. Gough, R. Vafabakhsh and R. Golestanian. Phys. Rev. Lett. 99, 048102 (2007).

[7] D. Bray, Cell Movements, Garland, New York (1992).

[8] M.J. Lighhill, Comm. Pure Appl. Math. 5109 (1952).

[9] J.R. Blake, J. Fluid. Mech 46, 199 (1971).

[10] S.R. De Groot and P. Mazur, Non-Equilibrium Thermodynamics, Dover (1984) 
[11] L. Landau and E.M. Lifschitz, Fluid Mechanics, Butterworth-Heinemann (1987).

[12] P.C. Martin, O. Parodi and P.S. Pershan, Phys. Rev. A 6, 2401 (1972).

[13] U. M. Córdova-Figueroa and J. F. Brady, Phys. Rev. Lett. 100, 4 (2008).

[14] J.-F. Joanny, F. Jülicher, K. Kruse, and J. Prost, New J. Phys. (2007).

[15] J. Happel and H. Brenner, Low Reynolds Number Hydrodynamics, Springer (1983).

[16] H.A. Stone and A.D.T Samuel, Phys. Rev. Lett. 77, 4102 (1996).

[17] D. Long and A. Ajdari, Phys. Rev. Lett. 81, 1529 (1998).

[18] J.R. Blake, Proc. Camb. Phil. Soc. 70, 303 (1971). 